Williams, ${ }^{4}$ angiographic studies were adopted and showed that the bleeding emerged from multiple sites, the major supplying vessels were then embolized, and the tumor was smoothly resected 3 days after embolization. However, in the case reported by Rao, Affifi, and Ghazarian, ${ }^{5}$ the patient was operated on without angiography, there was no single bleeding source and vascular abnormality identified intraoperatively, and surgical debridement and hemostasis were performed multiple times over the course of 26 days.

Such spontaneous hemorrhage is always insidious and minute with no apparent cause when it first begins. The diagnosis is often delayed, and the patients are in unavoidable and unstable hemodynamic status when they arrive at the hospital. Consequently, for emergency management, besides basic life support and the exclusion of coagulation abnormalities, the identification of the bleeding source should be the primary concern. Angiography coupled with subcutaneous embolization under DSA is a prompt, minimally invasive method for finding and controlling the bleeding vessels simultaneously, and it creates the possibility and enough preparation for later surgical intervention. One possible management strategy might be to consider routine surveillance of vascular lesions by angiography in patients with NF1, because it may be helpful in detecting and handling abnormal vessels earlier and thus prevent possible spontaneous hemorrhage.

Embolization is used to find the supplying vessels and control bleeding temporarily, but it cannot replace surgery. Although surgery is more aggressive and complicated owing to vessel fragility, it has a unique role in such cases: (1) it may be the only way and the best option for the permanent control of the ongoing or potential spontaneous hemorrhage; (2) early surgical resection of a huge tumor may reduce the possibility of malignant degeneration and of secondary skin necrosis and ulcerations caused by local malnutrition; and (3) surgery also has the potential for considerable positive cosmetic and pulmonary physiology impact. However, it is impossible to surgically remove all neurofibromas, which may affect virtually every nerve within the chest wall.

In conclusion, massive spontaneous hemorrhage in the soft tissue of the chest wall is very uncommon in patients with NF1. This case shows that angiography and embolization under DSA accompanied by surgery have great potential in the diagnosis and control of massive hemorrhage for patients with NF1.

We express our appreciation to Dr Jean Deslauriers (from Laval University, Canada) for his help in reviewing this case report.

\section{References}

1. Neurofibromatosis. Conference statement. National Institutes of Health Consen sus Development Conference. Arch Neurol. 1988;45:575-8.

2. Conlon NP, Redmond KC, Celi LA. Spontaneous hemothorax in a patient with neurofibromatosis type 1 and undiagnosed pheochromocytoma. Ann Thorac Surg. 2007;84:1021-3

3. Jett K, Friedman JM. Clinical and genetic aspects of neurofibromatosis 1. Genet Med. 2010;12:1-11.

4. Lessard L, Izadpanah A, Williams HB. Giant thoracic neurofibromatosis type 1 with massive intratumoral haemorrhage: a case report. J Plast Reconstr Aesthet Surg. 2009;62:e325-9.

5. Rao V, Affifi RA, Ghazarian D. Massive subcutaneous hemorrhage in a chest-wal neurofibroma. Can J Surg. 2000;43:459-60.

\title{
True aneurysm of anterior mitral leaflet-A rare entity
}

\author{
Trushar P. Gajjar, DNB, and Neelam B. Desai, MCh, DNB, Andhra Pradesh, India
}

True aneurysms, or diverticula, of the mitral valve are rare. $^{1,2}$ They are most common in patients with infective endocarditis affecting the aortic valve, but they can also present with chronic mitral regurgitation or as a complication of mitral valve prolapse. ${ }^{1,2}$ We present

\footnotetext{
From the Sri SathyaSai Institute of Higher Medical Sciences, Prasanthigram, District Anantapur, Andhra Pradesh, India.

Disclosures: Authors have nothing to disclose with regard to commercial support.

Received for publication March 23, 2012; revisions received April 20, 2012; accepted for publication May 16, 2012; available ahead of print June 18, 2012.

Address for reprints: Trushar P. Gajjar, DNB, Sri SathyaSai Institute of Higher Medical Sciences, Prasanthigram, District Anantapur, Andhra Pradesh 515134, India (E-mail: trushargajjar@gmail.com).

J Thorac Cardiovasc Surg 2012;144:e93-5

$0022-5223 / \$ 36.00$

Copyright (c) 2012 by The American Association for Thoracic Surgery

http://dx.doi.org/10.1016/j.jtcvs.2012.05.055
}

a case of true aneurysm of anterior mitral leaflet causing mitral regurgitation and describes the pathogenesis, diagnostic modalities, and surgical decision with a review of the published data.

\section{CASE REPORT}

A 39-year-old man presented with palpitation and breathlessness on exertion, New York Health Association class II. Physical examination revealed a pansystolic murmur at the apex radiating posteriorly up to the midscapular line. Chest radiography showed a cardiothoracic ratio of $60 \%$ to $65 \%$ and pulmonary hilar congestion. The electrocardiogram showed sinus rhythm, normal axis, and left ventricular hypertrophy. The transthoracic and transesophageal echocardiogram showed an aneurysm of the anterior mitral leaflet 

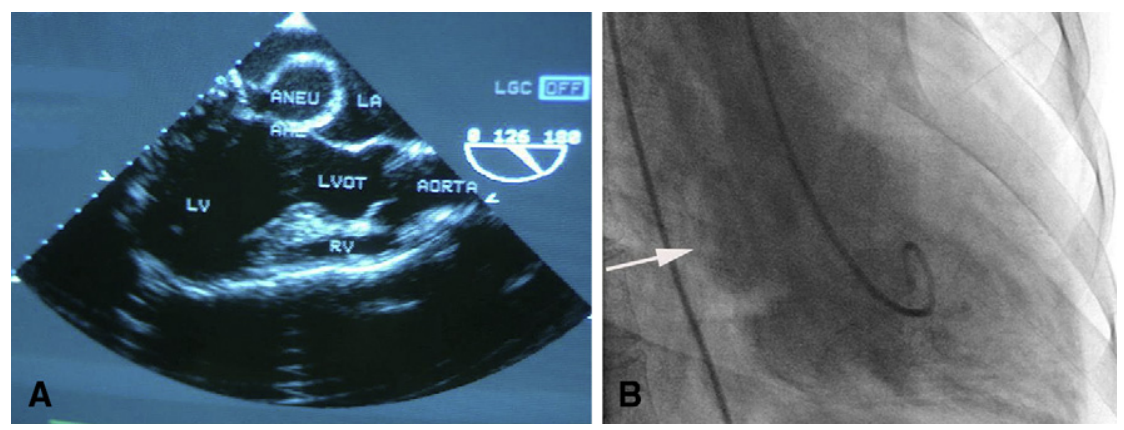

FIGURE 1. A, Transesophageal echocardiogram showing an aneurysm of the anterior mitral leaflet with the mouth of the aneurysm in communication with the left ventricle (LV). B, Left ventriculogram in right anterior oblique view showing aneurysm bulging from mitral valve (arrow). AML, Anterior mitral leaflet; $P M L$, posterior mitral leaflet; $L A A$, left atrial appendage; $L A$, left atrium; $R V$, right ventricle; $L V O T$, left ventricular outflow tract.

near the posteromedial commissure causing moderate to severe mitral regurgitation. The subvalvar apparatus and remaining areas of the mitral valve leaflet appeared normal. The other valves appeared to be normal (Figure 1,A). Left ventriculogram in the right anterior oblique (Figure 1,B) and 4-chamber view showed anterior mitral leaflet aneurysm with severe mitral regurgitation. The white blood cell and platelet counts were normal. The blood culture was negative. Intraoperatively, a $4 \times 4-\mathrm{cm}$, smooth, yellowish white dome-shaped uniformly thick-walled aneurysm of the anterior mitral leaflet was found, located more toward the posteromedial commissure (Figure 2, $A$ ). No clot or thrombus was found in the aneurysm cavity (Figure 2, $B$ ). The surrounding $1-\mathrm{cm}$ portion of the leaflet was thickened and distorted. The aneurysm occupied approximately half the leaflet. The posterior mitral leaflet, remaining anterior mitral leaflet, and subvalvar apparatus were normal. The aneurysm was excised, and repair was attempted but was unsuccessful. The mitral valve was excised and replaced with a mechanical valve. Histopathologic examination showed a $3.5 \times 3.2-\mathrm{cm}$, dome-shaped hollow specimen that was whitish yellow in color. Microscopically, the irregularly thickened aneurysm wall showed predominantly fibrocollagenous tissue with a few islands of basophilic degeneration and a mild vascularity suggestive of valvulitis.

\section{DISCUSSION}

A true aneurysm of the mitral valve is uncommon. ${ }^{1-3}$ Aneurysms of the mitral valve are most common in patients with infective endocarditis affecting the aortic valve, but they can also present with chronic mitral regurgitation or as a complication of mitral valve prolapse. ${ }^{2,3}$ Our patient did not have a history or clinical or laboratory evidence of infective endocarditis. A true aneurysm, when present, is usually found near the base of the anterior cusp. ${ }^{3}$ Saphir and Leroy, ${ }^{1}$ in 1948 , stressed the rarity of true aneurysms of the mitral valve and stressed that these types of aneurysms are definitely not "thromboaneurysms" or false aneurysms. They are the result of valvulitis with consequent formation of granulation tissue and scar tissue that succumbed to intraventricular pressure with the formation of sac-like outpouchings. ${ }^{1}$ The findings from our case matched the macroscopic and microscopic description of the true aneurysm given by Saphir and Leroy. ${ }^{1}$ The diagnosis of mitral aneurysm is difficult using transthoracic echocardiography, ${ }^{2}$ as reported in published studies; however, in the present case, the diagnosis was done primarily using transthoracic echocardiography, which might have been because it was a large and well-defined aneurysm. Transesophageal echocardiography is an important and reliable diagnostic modality that provides detailed anatomic
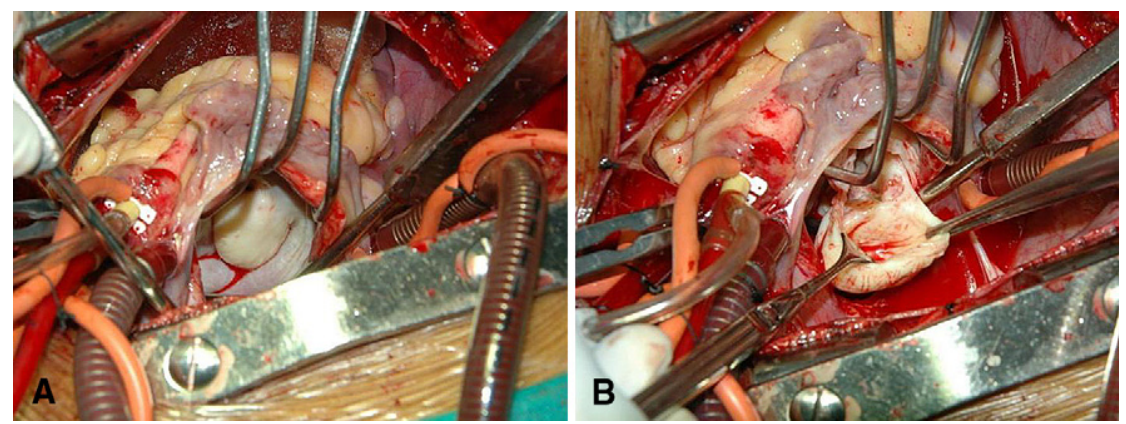

FIGURE 2. Intraoperative view through opened left atrium. A, Aneurysm of anterior mitral leaflet near posteromedial commissure. B, Intraoperative view showing partially cut opened aneurysm without clots or thrombus in the cavity. 
and morphologic view of the lesion. ${ }^{3}$ The angiocardiogram is not as important as the echocardiogram in delineating the aneurysm. ${ }^{2}$ In the present case, the left ventriculogram in the right anterior oblique and 4-chamber view delineated the mitral regurgitation and the aneurysm of the anterior leaflet. Intraoperatively, it is important to determine the precise pathologic anatomy. Depending on the nature and site of the mitral lesion, conservation of the mitral valve could be possible, especially if the aneurysm is small or located on the posterior mitral leaflet. ${ }^{3}$ However, with large aneurysm such as in the present case with nearly $50 \%$ leaflet involvement and involvement of the subvalvar apparatus, replacement of the valve is the only choice.

\section{CONCLUSIONS}

True aneurysm of the mitral valve is rare entity. Transesophageal echocardiography is an important noninvasive diagnostic modality. The decision to repair or replace the valve is mainly dependent on the size, the site of the aneurysm, the condition of the surrounding tissue, and subvalvar involvement.

\section{References}

1. Saphir O, Leroy EP. True aneurysms of the mitral valve in subacute bacterial endocarditis. Am J Pathol. 1948;24:83-95.

2. Enia F, Celona G, Filippone V. Echocardiographic detection of mitral valve aneurysm in patient with infective endocarditis. Br Heart J. 1983;49:98-100.

3. Northridge DB, Gnanapragasam JP, Houston AB. Diagnosis of mitral valve aneurysm by transoesophageal echocardiography. Br Heart J. 1991;65:227-8.

\title{
Nonintubated thoracoscopic surgery for pulmonary lesions in both lungs
}

\author{
Tung-Ming Tsai, MD, and Jin-Shing Chen, MD, PhD, Taipei, Taiwan
}

Thoracoscopic resections for pulmonary lesions in both lungs are usually performed under conditions of general anesthesia with double-lumen endotracheal intubation and sequential single-lung ventilation. ${ }^{1}$ A newly developed nonintubated technique that uses thoracic epidural anesthesia and sedation has been introduced for thoracoscopic resection of unilateral pulmonary lesions, with satisfactory results. ${ }^{2-5}$ Nonintubated thoracoscopic surgery for lesions in both lungs, however, has not previously been reported. Here we report a case of successful resection with a nonintubated thoracoscopic technique for bilateral pulmonary lesions. Our results indicate that nonintubated thoracoscopic surgery can be used in a specific group of patients.

\section{CLINICAL SUMMARY}

A 57-year-old nonsmoking woman was referred to our hospital for undetermined bilateral pulmonary lesions. She had a medical history of Sjögren syndrome complicated

\footnotetext{
From the Divisions of Experimental Surgery, and Thoracic Surgery, Department of Surgery, National Taiwan University Hospital and National Taiwan University College of Medicine, Taipei, Taiwan.

Disclosures: Authors have nothing to disclose with regard to commercial support.

Received for publication March 13, 2012; revisions received May 21, 2012; accepted for publication June 12, 2012; available ahead of print July 12, 2012.

Address for reprints: Jin-Shing Chen, MD, PhD, Division of Thoracic Surgery, Department of Surgery, National Taiwan University Hospital, No. 7, Chung Shan S Rd, Taipei, Taiwan (E-mail: chenjs@ntu.edu.tw).

J Thorac Cardiovasc Surg 2012;144:e95-7

0022-5223/\$36.00

Copyright (C) 2012 by The American Association for Thoracic Surgery

http://dx.doi.org/10.1016/j.jtcvs.2012.06.025
}

with xerostomia for more than 10 years. Serial thoracic computed tomographic scans showed 2 ground-glass opacities: 1 corresponded to a $0.5-\mathrm{cm}$ previously undetected lesion in the right lower lobe, and the other corresponded to a $0.7-\mathrm{cm}$ stationary lesion in the left lower lobe (Figure 1). The patient had a family history of lung cancer, and computed tomographic findings suggested the presence of multiple early lung cancers. The preoperative forced expiratory volume in 1 second was $122 \%$ of the prediction value. After discussion with our surgical team, the patient opted for nonintubated anesthesia during thoracoscopic resection to avoid the airway mucosal injury caused by severe xerostomia after endotracheal intubation.

In the operating room, the patient was medicated before surgery with an intravenous infusion of $50 \mu \mathrm{g}$ fentanyl. Thoracic epidural anesthesia was administered in the T5-6 thoracic interspace by continuous infusion of $2 \%$ lidocaine. The patient was then sedated with an intravenous infusion of propofol $(10 \mathrm{mg} / \mathrm{mL})$, with a target-controlled infusion method used to maintain her in a mildly sedated, but communicative and cooperative, state (Ramsay sedation score III). During the procedure, the patient breathed oxygen through a ventilation mask.

Subsequently, bilateral sequential thoracoscopic surgery was performed with the patient in the left decubitus position. The right pulmonary lesion was identified by preoperative computed tomographically guided hookwire localization. During right thoracoscopy, the right lung collapsed gradually under spontaneous breathing. To inhibit coughing during thoracoscopic manipulation, a vagal block 